\title{
USO DA AVALIAÇÃO DE IMAGENS ASSISTIDA POR COMPUTADOR (CAIA) NA \\ DETECÇÃO DE Ki67 EM CASOS DE CÂNCER DE MAMA - REVISÃO DA \\ LITERATURA
}

\section{Categoria Clínico}

INSTITUIÇÃO: Centro Universitário São Camilo.

AUTORES: Bottura HR ${ }^{1}$;

Rua Humberto I 962, Vila Mariana - São Paulo (SP)- 04018.033; (17) 991388556; e-mail: heeitorbottura@hotmail.com;

Molina $\mathrm{CA}^{1}$;

ORIENTADORES: Raia DA².

Discentes da Faculdade de Medicina do Centro Universitário São Camilo ${ }^{\text {; }}$ Médica dermatologista pela Faculdade de Medicina de Rio Preto - FAMERP. ${ }^{2}$ 
USO DA AVALIAÇÃO DE IMAGENS ASSISTIDA POR COMPUTADOR (CAIA) NA DETECÇÃO DE Ki67 EM CASOS DE CÂNCER DE MAMA - REVISÃO DA LITERATURA

Categoria Clínico

DESCRITORES: “COMPUTER-AIDED DIAGNOSIS”; "KI67”; "BREAST CÂNCER 


\section{RESUMO}

INTRODUÇÃO: O manejo de casos de câncer de mama demanda a avaliação de marcadores biológicos, como o Ki67, utilizado para mensurar a dimensão de mitoses do tecido neoplásico. Por meio de exames imuno-histoquímicos é necessária a presença de um profissional capacitado para essa avaliação, contudo, com a evolução da robótica, uma das estratégias cada vez mais empregadas é a avaliação de imagens assistida por computador (CAIA). Esta técnica promete realizar o trabalho de identificação de padrões, reconhecendo o extrato anatomopatológico e realizando o diagnóstico do caso.

OBJETIVOS: Buscar na literatura relatos da eficácia da técnica de CAIA na identificação imunohistoquímica do marcador Ki67 em casos de câncer de mama.

METODOLOGIA: Realizada pesquisa no banco de dados PubMed, utilizando-se os descritores: "computer-aided diagnosis", "Ki67" e "breast câncer" e os critérios de inclusão "publicação nos últimos 5 anos" e "idioma". Um total de 14 artigos foram encontrados e 8 foram selecionados de acordo com os critérios de exclusão "estudos secundários" e "não adequação ao objetivo".

RESULTADOS: Os estudos analisados compararam a detecção de Ki67 pelo método visual (profissional capacitado) e por meio da avaliação de imagens assistida por computador (CAIA). Em relação a detecção do local de interesse observou-se uma assertividade de $82 \%$ da CAIA em relação a mesma avaliação realizada por profissionais. Essa taxa permite considerar o método estatisticamente eficaz na identificação de padrões de expressão do Ki67. Nesse âmbito, a CAIA demonstrou maior acurácia na identificação dos padrões baixo (Ki67 $\leq 10 \%$ ) e moderado $(10 \%<\mathrm{Ki} 67<50 \%)$, quando comparado ao método visual. Poucas discordâncias ocorreram entre as duas avaliações, principalmente relacionadas a heterogeneidade dos tumores. Essas comparações evidenciam a CAIA como um método eficiente e rápido (média de 54 segundos por imagem) com potencial para otimizar esse tipo de avaliação.

CONCLUSÃO: A avaliação de marcadores biológicos como o Ki67 é etapa essencial para consolidação de um plano terapêutico e prognóstico nos casos de câncer de mama. Nesse cenário, a CAIA tem se firmado como um método de maior rapidez e semelhante acurácia quando comparado a detecção dos locais de interesse pelo método visual. Desse modo, esse avanço tecnológico configura-se como uma interessante ferramenta de suporte, visando aperfeiçoar as técnicas convencionais. 


\section{USO DA AVALIAÇÃO DE IMAGENS ASSISTIDA POR COMPUTADOR (CAIA) NA DETECÇÃO DE Ki67 EM CASOS DE CÂNCER DE MAMA - REVISÃO DA LITERATURA}

\section{INTRODUÇÃO}

O câncer de mama é uma doença heterogênea e multifatorial, logo, seu manejo demanda uma série de análises que potencializam a avaliação prognóstica dos pacientes. Diante disso, a tomada de decisão terapêutica no câncer de mama depende não apenas dos aspectos clínicos, mas também da análise de biomarcadores histopatológicos, como o índice de proliferação do Ki67 ${ }^{(1)}$.

Ki67 é um antígeno nuclear que é expresso durante as fases, S, G2 e M do ciclo celular, bem como na fase G1, logo após a mitose. Logo, somente não é expresso nas fases G0 e pouco após a transição de G0 e G1. Sua principal utilização é como variável para avaliar (i) o prognóstico do câncer de mama, (ii) possível resposta à hormonioterapia neoadjuvante e (iii) calcular os benefícios da quimioterapia adjuvante ${ }^{(1)}$.

A identificação de hotspots, ou seja, aréas com características histopatológicas positivas para o tipo de lesão procurada, bem como a quantificação imuno-histoquímica do Ki67, são essenciais para avaliação prognóstica e para o planejamento terapêutico no câncer de mama. Uma alta porcentagem de positividade de Ki67 dentro de uma biópsia indica uma maior taxa de proliferação e, consequentemente, maior agressividade de um tumor ${ }^{(2)}$. Essa taxa de proliferação é normalmente estimada visualmente pelo patologista (método de análise visual - AV) em uma área de hotspot que deve incluir de 500 a 2000 células $^{(2)}$.

Em relação a detecção do Ki67, a avaliação imunohistoquímica da proporção de células marcadas com o antígeno nuclear é o método mais utilizado em amostras clínicas. Todavia, esse método apresenta algumas desvantagens, como suscetibilidade a fatores interferentes, alta discrepância entre laboratórios e interpretação subjetiva, limitando assim severamente sua utilidade ${ }^{(3)}$. Essa subjetividade evidencia-se principalmente na ocorrência de tumores com características heterogênias, nos quais a categorização da expressão do biomarcador é bastante dificultada.

Diante disso, o aperfeiçoamento de novas tecnologias aparece como um potencializador dessas análises. Nesse contexto, outro método de diagnóstico armado é uso de pontos quânticos (QD), tecnologia utilizada para aumentar a eficácia da imunohistoquímica na detecção da expressão de Ki67. Os pontos quânticos (QDs), são um novo nanocristal semicondutor com propriedades fotofísicas particulares, como bandas de emissão simétricas ajustáveis pelo tamanho, absorbância de luz superior, alta intensidade fluorescente e fotoestabilidade forte ${ }^{(3)}$. 
Ademais, com a evolução cada vez mais significativa da robótica e computação destaca-se um novo recurso capaz de, entre outras muitas funções, otimizar a detecção e avaliação de biomarcadores: o método de avaliação de imagens assistida por computador (CAIA). Neste, um software é desenvolvido de modo a processar e analisar imagens histopatológicas, chegando a um diagnóstico, avaliação, quantificação ou categorização independente ou parcialmente independente de um profissional operador.

Diferentes métodos computadorizados já foram utilizados como alternativa na detecção de Ki67. Um dos mais recentes trata-se de uma contagem automática, considerada um método alternativo promissor, uma vez que, em oposição a outros softwares já testados, é capaz de reconhecer e contar células cancerígenas tanto Ki67 positivas quanto negativas, tornando-se assim uma avaliação mais objetiva, eficiente e precisa ${ }^{(7)}$.

Os diferentes métodos de CAIA aparecem como alternativas promissoras diante de suas propriedades de objetividade e rapidez, além de valor prognóstico mais forte que a contagem manual. No entanto, a precisão desses métodos também é questionada levando em consideração a dificuldade de desenvolver algoritmos sensíveis a tantas variáveis quanto as presentes em imagens histopatológicas $^{(7)}$. Isto é, o reconhecimento computadorizado de Ki67 em células cancerígenas baseia-se, essencialmente, nas características de seus núcleos tais como cor, forma e tamanho, contudo é difícil eliminar interferências de células não cancerosas do estroma (como células linfocitárias, fibroblastos, macrófagos) $)^{(7)}$.

\section{OBJETIVOS}

\subsection{OBJETIVO GERAL}

- Buscar na literatura relatos da eficácia da técnica de avaliação de imagens assistida por computador na identificação imunohistoquímica do marcador Ki67 em casos de câncer de mama.

\subsection{OBJETIVOS ESPECÍFICOS}

- Identificar as finalidades e importância da detecção de biomarcadores, em especial Ki67, no manejo dos pacientes com câncer de mama.

- Identificar as técnicas de detecção de Ki67 mais utilizadas e suas respectivas características.

- Buscar quais as novas técnicas na detecção de Ki67 e suas vantagens.

- Avaliar a eficácia da técnica de avaliação de imagens assistida por computador em comparação com a avaliação visual na identificação imunohistoquímica do marcador Ki67 em casos de câncer de mama. 


\section{METODOLOGIA}

O estudo foi desenvolvido por meio de uma revisão da literatura utilizando-se a base de dados PubMed. Com os descritores "computer-aided diagnosis", "Ki67" e "breast cancer" foram encontrados um total de 14 estudos e selecionados 8. Os critérios de exclusão adotados foram “estudos secundários" e "não adequação ao objetivo". (Fig.1)

\section{PESQUISA NA BASE DE DADOS PubMED}

"computer-aided diagnosis AND Ki67 AND breast cancer"

14 ESTUDOS ENCONTRADOS

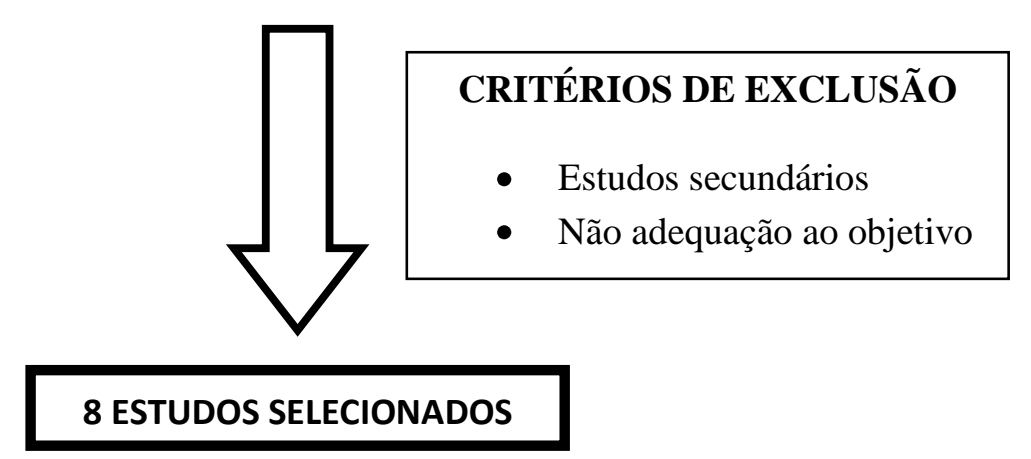

Fig.1. Fluxograma de metodologia.

\section{RESULTADOS}

Nos trabalhos analisados, os resultados foram obtidos através da comparação entre a análise visual (AV), composta pelo método tradicional de avaliação microscópica por um profissional patologista capacitado, e o método de avaliação de imagens assistida por computador (CAIA), onde um software processa e analisa a imagem, resultando em um diagnóstico independente (ou parcialmente independente) do profissional operador.

Em geral, os resultados obtidos na avaliação da literatura disponível são otimistas. Em relação à detecção automática de hotspots (AKHoD), Pilutti et al (2017) constatou um reconhecimento 
correto de $81.94 \%$ dos hotspots em 72 peças imunohistoquímicas, sendo o padrão-ouro a comparação com três profissionais de decisão unanime. Levando em consideração o consenso entre apenas dois dos três operadores, a AKHoD obteve uma taxa de acertos de $78.50 \%$ e, quando comparado com a opinião isolada de apenas um patologista, $60.61 \%$. Alguns pontos sugeridos pelo AKHoD não foram observados pela análise visual, destacando a utilidade prática do software em questão, capaz de sugerir a área como uma candidata para avaliação adicional pelo observador ${ }^{(2)}$.

No âmbito da avaliação quantitativa da expressão do KI67, os estudos são concordantes em afirmar uma boa avaliação realizada por parte do CAIA. A coorte de Zhong et. al (2016) demonstra que o Coeficiente de Correlação Intraclasse (ICC) entre a AV e a CAIA é de 0.974, ou seja, ambos os métodos mantém uma concordância quase perfeita entre seus resultados ${ }^{(4)}$.

Destes, o maior grau de discordância (ainda relativamente baixo), foi encontrado nas expressões moderadas $(11 \%<$ Ki67 < 30\%) de Ki67. Nas expressões baixas (Ki67<10\%) e elevadas (>30\%) foram encontrados os maiores graus de consonância. Ainda, a heterogeneidade do tumor também foi constatada como um fator que pode alterar negativamente a concordância entre AV e CAIA. ${ }^{(4)} \mathrm{O}$ estudo ainda mostra que os resultados obtidos pela AV eram consistentemente mais baixos quando comparados aos resultados da CAIA. Por fim, o estudo corrobora com o trabalho de Piluttiet al (2017), mostrando um ICC de 0.957 entre a detecção de hotpots por VA e CAIA ${ }^{(4) .}$

Nos trabalhos de Laurinaviciuset al (2014 e 2016), fica claro a competência do método de diagnóstico digital na caracterização da expressão do KI67 e sua consequente previsão de mortalidade e sobrevivência. Neles, o autor reforça que, tanto no método de AV quanto em CAIA, os níveis de expressão do Ki67 forneceram valores de corte significativos como preditores isolados de sobrevivência nos casos de câncer de mama ${ }^{(4,5)}$.

Ademais, outro método de diagnóstico armado é uso de pontos quânticos (QD) para aumentar a eficácia da imunohistoquímica na detecção da expressão de Ki67, melhorando a qualidade da imagem e a sensibilidade do exame. O estudo de Wang et al (2016) desenvolveu um método eficaz de medição de Ki67 por análise digital de imagem baseado no método de pontos quânticos, de forma que o ICC entre o padrão ouro de diagnóstico e o método computadorizado foi calculado em 0.964 , sendo maior quando comparado ao método de avaliação visual subjetiva por operantes (ICC=0.923). Nesse âmbito, a CAIA demonstrou sua melhor acurácia na identificação dos padrões baixo $($ Ki67 $\leq 10 \%)$ e moderado $(10 \%<$ Ki67 $<50 \%)$, quando comparado ao método visual ${ }^{(6)}$.

Outros estudos ainda enfatizam a detecção automática digital como um método de baixo custo e alta velocidade para a mensuração da expressão de Ki67, auxiliando, portanto, no diagnóstico do 
subtipo tumoral ${ }^{(8)}$. É documentada a velocidade média de detecção de hotspots de 54 segundos por peça anatomopatológica ${ }^{(4)}$.

\section{CONCLUSÕES}

Em geral, uma boa concordância entre os resultados obtidos pela análise visual (AV) e pela avaliação de imagens assistida por computador (CAIA) foi descrita em todos os estudos analisados, sugerindo que o método digital poderia ser utilizado como ferramenta auxiliadora para avaliar o grau de expressão do Ki67. Tanto a detecção de hotspots quanto a avaliação quantitativa da expressão do Ki67 são procedimentos que consomem tempo e dependem da experiência do profissional operador.

Assim, os softwares de detecção de imagem são ferramentas capazes de ajudar o observador, sugerindo áreas de análise na peça anatomopatológica e corroborando para uma hipótese diagnóstica mais precisa.

\section{REFERÊNCIAS}

1. The region-of-interest size impacts on Ki67 quantification by computer-assisted image analysis in breast cancer Matthias Christgen MD, PhD $\square$, Sabrina von Ahsen, Henriette Christgen, Florian Länger MD, Hans Kreipe MD

2. Pilutti D, Della Mea V, Pegolo E, La Marra F, Antoniazzi F, Di Loreto C. An adaptive positivity thresholding method for automated Ki67 hotspot detection (AKHoD) in breast cancer biopsies. Comput Med Imaging Graph. novembro de 2017;61:28-34.

3. Sun S-R, Sun J-Z, Chen C, Jiang G, Li Y, Tian W-Q. Quantum dot-based immunofluorescent imaging of Ki67 and identification of prognostic value in HER2-positive (non-luminal) breast cancer. Int J Nanomedicine. março de 2014;1339.

4. Zhong F, Bi R, Yu B, Yang F, Yang W, Shui R. A Comparison of Visual Assessment and Automated Digital Image Analysis of Ki67 Labeling Index in Breast Cancer. Coppola D, organizador. PLOS ONE. 29 de fevereiro de 2016;11(2):e0150505.

5. Laurinavicius A, Plancoulaine B, Rasmusson A, Besusparis J, Augulis R, Meskauskas R, et al. Bimodality of intratumor Ki67 expression is an independent prognostic factor of overall survival in patients with invasive breast carcinoma. Virchows Arch. abril de 2016;468(4):493502.

6. Laurinavicius A, Plancoulaine B, Laurinaviciene A, Herlin P, Meskauskas R, Baltrusaityte I, et al. A methodology to ensure and improve accuracy of Ki67 labelling index estimation by automated digital image analysis in breast cancer tissue. Breast Cancer Res [Internet]. abril de 2014 [citado 22 de setembro de 2018];16(2). Disponível em: http://breast-cancerresearch.biomedcentral.com/articles/10.1186/bcr3639

7. Wang L-W, Qu A-P, Liu W-L, Chen J-M, Yuan J-P, Wu H, et al. Quantum dots-based double imaging combined with organic dye imaging to establish an automatic computerized method for cancer Ki67 measurement. Sci Rep [Internet]. agosto de 2016 [citado 22 de setembro de 2018];6(1). Disponível em: http://www.nature.com/articles/srep20564 
8. Gándara-Cortes M, Vázquez-Boquete Á, Fernández-Rodríguez B, Viaño P, Ínsua D, SeoaneSeoane A, et al. Breast cancer subtype discrimination using standardized 4-IHC and digital image analysis. Virchows Arch. fevereiro de 2018;472(2):195-203. 